\title{
DOENÇAS SEXUALMENTE TRANSMISSÍVEIS
}

\section{PAPILOMAVIRUS HUMANO E O CÂNCER ANAL}

\author{
SIDNEY ROBERTO NADAL ${ }^{1}$, CARMEN RUTH MANZIONE $^{1}$
}

${ }^{1}$ Instituto de Infectologia do Hospital Emilio Ribas, São Paulo

NADAL SR, MANZIONE CR. Papilomavirus Humano e o Câncer Anal. Rev bras Coloproct, 2006;26(2):204-207

RESUMO: O Papilomavirus humano (HPV) é uma das causas mais comuns de doença sexualmente transmissível, podendo provocar os condilomas acuminados que são considerados fatores de risco para displasia e neoplasia. Embora os HPV de alto risco sejam causa necessária para o câncer cervical, eventos genéticos adicionais são indispensáveis para transformação maligna da maioria dos carcinomas anais e de outros sítios. Os trabalhos da literatura especializada ainda não conseguiram demonstrar se esse vírus é o fato determinante ou associado ao carcinoma anal. É preciso que mais pesquisas sejam feitas para resolver esse dilema. De qualquer forma, sugerimos que o controle das lesões clínicas e das sub-clínicas provocadas pelo HPV possa evitar a eventual progressão para carcinoma invasivo.

Descritores: Infecções por Papilllomavirus. Neoplasia intra-epitelial; prevenção e controle. Canal anal; citologia. Carcinoma de células escamosas.

\section{INTRODUÇÃO}

O Papilomavirus humano (HPV) é uma das causas mais comuns de doença sexualmente transmissível, podendo provocar os condilomas acuminados que são considerados fatores de risco para displasia. ${ }^{1}$ Essa, que vem sendo chamada de neoplasia intraepitelial anal (NIA), é conseqüência da infecção crônica pelo HPV na região perianal e parece ser causada pela carga viral elevada. ${ }^{2,3}$

Embora a história natural dessa lesão seja incerta, ${ }^{4,5}$ acredita-se que a NIA seja precursora do carcinoma anal. ${ }^{16}$ Entretanto, a NIAA (de alto grau) parece ter baixo potencial para transformação maligna em doentes imunocompetentes e se espera que tenha incidência aumentada em imunodeprimidos, bem como determine mais alteração neoplásica. ${ }^{5}$

Sua incidência permanece elevada entre os homens HIV-positivos que praticam sexo com homens, mesmo após a introdução dos esquemas com drogas anti-retrovirais (ARV). ${ }^{2,3}$ Apesar da melhora da imunidade conferida por esse tratamento, o número de NIAA mantém-se similar ao período em que os inibidores da protease não eram disponíveis, ${ }^{7}$ indicando a necessidade de prevenção do câncer anal nesses doentes, estejam ou não usando essa medicação., ${ }^{2,3}$

Nos homens que fazem sexo com homens (HSH) e com múltiplos parceiros sexuais, a prevalência

Trabalho realizado pela Equipe técnica de Proctologia do Instituto de Infectologia do Hospital Emilio Ribas, São Paulo

Recebido em 08/05/2006

Aceito para publicação em 04/06/2006 
do HPV não diminui com a idade, diferente do observado nos doentes heterossexuais. A NIA é igualmente prevalente em grupos com diferentes idades entre os HSH, mas por outro lado sabe-se que a história natural é semelhante à da neoplasia intraepitelial cervical. Sendo assim, as lesões de baixo grau freqüentemente se resolvem, enquanto as de alto grau são mais estáveis. Doentes HIV-positivos que praticam sexo anal receptivo são os de maior risco para NIA. ${ }^{8}$ Já os HSH HIVnegativos têm alta prevalência de NIA, independendo da faixa etária, o que reflete sua exposição sexual ao HPV. Numa análise multivariada, o risco para NIAB (de baixo grau) foi associado a ter mais que cinco parceiros sexuais, uso de drogas injetáveis duas ou mais vezes por mês nos últimos seis meses, idade mais avançada para o primeiro contato anal receptivo e infecção por muitos HPV associados. Todavia, o risco para NIAA esteve associado com qualquer lesão pelo HPV e com associação dos vários tipos virais. ${ }^{9}$

Em estudo anterior, observamos a presença de NIAA em $18,56 \%$ dos nossos doentes. Nenhum deles evoluiu para CEC invasivo em seguimento de 6 a 12 anos, embora a recidiva dos condilomas ocorresse mais naqueles com NIAA que com NIAB, principalmente nos estádios mais avançados da infecção pelo HIV. Todavia, os doentes com NIAA não reincidiram com o mesmo padrão histológico. Desta forma, concluímos que a NIAA e os estádios mais tardios da AIDS foram os fatores de risco para recidiva dos condilomas acuminados anais, mas não para sua evolução para carcinoma invasivo. ${ }^{10}$

O principal fator de desenvolvimento do carcinoma espinocelular (CEC) anal invasivo em doentes com HIV parece ser o tempo de infecção por esse vírus. Como há aumento do tempo de vida secundário à terapia ARV, induz-se que esse tumor se tornará mais frequiente.$^{4,11}$ Outros fatores, como o tabagismo, o sexo anal receptivo, infecção pelo HPV e o número de parceiros sexuais durante a vida, podem propiciar a incidência aumentada de câncer anal em homens e mulheres. ${ }^{12}$ Acreditamos que a gênese tumoral esteja no tripé formado pelo fator viral e pelas imunidades local e sistêmica, uma vez que o tipo viral parece ser importante e a imunodepressão prolongada está associada à freqüência aumentada de tumores cervicais e anais. ${ }^{3,13,14}$

O DNA do HPV pôde ser detectado pelo PCR (reação em cadeia da polimerase) em até $85,6 \%$ dos exames em caso de NIAA, ${ }^{15,16}$ sendo o tipo 16 o mais comum, ${ }^{15}$ e em até 47,4\% das NIAB. ${ }^{16}$ Já os aspectos histológicos do HPV foram observados em todos os doentes. ${ }^{15}$ Em relação à captura híbrida, o vírus foi identificado em $35,2 \%$ das lesões penianas sugestivas de infecção pelo HPV nas biópsias. ${ }^{17}$ E quando os dois métodos foram associados observou-se $54 \%$ de sensibilidade nas lesões cervicais. ${ }^{18}$ Todavia, usando o PCR, constatamos os HPV 6 e 11 nos casos de NIAA, o que nos permitiu concluir que tanto os tipos oncogênicos como os não oncogenicos podem estar associados com NIAA nos doentes HIV-positivos. ${ }^{19}$ Esse fato sugere que os imunodeprimidos com lesões provocadas pelo HPV devam ser seguidos criteriosamente, independendo do tipo do HPV. Além disso, a presença de múltiplos tipos virais, incluindo os de alto risco, é comum nas verrugas anogenitais, ${ }^{20} \mathrm{e}$ os métodos de detecção do DNA do HPV são menos efetivos que a biópsia dirigida para diagnóstico da doença. ${ }^{15,18}$ Desta forma, parecem ser mais importantes para confirmar o diagnóstico histológico do que informar o padrão de oncogenicidade.

A citologia anal isolada não tem acurácia para detectar lesões de alto grau em doentes com condilomas anais, com sensibilidade de $42 \%$ e especificidade de 96\%. ${ }^{1}$ Entretanto, observamos sensibilidade de $74 \%$ e especificidade de $61 \%$ com o método. ${ }^{21}$ Como outros autores relataram sensibilidade entre 69 e $98 \%$ e de especificidade entre 16,3 e $50 \%,{ }^{1,8,22-26}$ acreditamos que a técnica de coleta seja responsável pelas diferenças entre esses índices.

Não há tratamento que erradique satisfatoriamente as lesões pré-malignas com baixa morbidez e pouca recidiva. A excisão extensa da displasia tem alta morbidez e resultado questionável e outros tratamentos têm valor incerto. Como a maioria dos doentes acompanhados não desenvolveu carcinoma invasivo e naqueles que o tiveram o tumor foi identificado no estádio inicial, o mesmo pôde ser curado. ${ }^{27} \mathrm{O}$ rastreamento com exame físico para carcinoma invasivo pode ser aceitável para seguimento de doentes HIV-positivos com biópsias que demonstrem NIA. ${ }^{27}$

Os mecanismos moleculares envolvidos na progressão do carcinoma espinocelular anal são pouco elucidados, bem como a função da infecção pelo HIV. A perda da heterozigosidade ( $\mathrm{LOH})$ é um dos mecanismos responsáveis pela inativação dos genes supressores de tumor. ${ }^{13}$ Dados atuais sugerem que 
mutações nos genes supressores de tumor p53, DCC e APC contribuem para a evolução para CEC anal invasivo em indivíduos imunocompetentes. Entre os doentes HIV-positivos a infecção persistente pelo HPV no canal anal é mais comum. Nessa população, a instabilidade dos microssatélites, mais que a instabilidade cromosômica, parece ser o fator mais importante de progressão para carcinoma invasivo. ${ }^{8}$ A transformação das células epiteliais humanas mediadas pelo HPV tem sido reconhecida, resultando de transcripção desregulada dos oncogenes virais E6 e E7 nas células em proliferação. A interferência do E6 e do E7 com o ciclo celular induz instabilidade genética, produzindo células com alterações oncogênicas com fenótipo maligno. ${ }^{29}$ Eventos genéticos iniciais durante a carcinogênese cervical associadas com imortalização, incluem deleção dos cromossomos 3q, 6 e 10p, enquanto que entre outros, ganhos no cromossomo 3q, perda no 11 e alteração epigenética como inativação do gene supressor de tumor TSLC1 representa eventos tardios associados com a invasão do tumor. ${ }^{29}$ Além disso, observou-se que os tumores dos doentes HIV-negativos apresentaram mais LOH que nos soropositivos. LOH nos cromossomos 17p, 18q, 5q, e 11q foram notados em doentes HIV-negativos com CEC anal. Em contraste, as perdas dos alelos em 17p, 5q, e 18q parecem ser raras em tumores dos indivíduos HIV-positivos. ${ }^{28}$ Outros autores observaram ganhos nos cromossomos 1p, 1q, $3 q, 8 p$ e 20q, e perdas nos 2q, 7q, 11p, 11q e 15q, que poderiam ativar vários oncogenes. ${ }^{6}$ Esses dados sugerem que a imunodepressão pode promover a progressão para CEC através de um padrão alternativo e que a persistência da infecção pelo HPV no canal anal desempenhe a função central nesse processo. ${ }^{13}$

A infecção pelo HPV e a subseqüente integração do DNA do HPV são necessárias, mas não suficientes para causar progressão para câncer. ${ }^{18}$ Embora os HPV de alto risco sejam causa necessária para o câncer cervical, eventos genéticos adicionais são indispensáveis para a transformação maligna da maioria dos carcinomas anais e de outros sítios. ${ }^{29}$ Os trabalhos da literatura especializada ainda não conseguiram demonstrar se o HPV é o fator determinante ou associado ao carcinoma anal. Acreditamos no anseio da comunidade científica de ligar o câncer a um fator etiológico que removido evitaria ou trataria a doença. É preciso que mais pesquisas sejam feitas para resolver esse dilema. De qualquer forma, sugerimos que o controle das lesões clínicas e sub-clínicas provocadas pelo HPV evita a eventual progressão para carcinoma invasivo.

SUMMARY: Human Papillomavirus (HPV) is one of the commonest sexually transmitted diseases agents. It can provoke condylomata acuminata, considered at risk to dysplasia and neoplasia. Although, high-risk HPVs are necessary to cervical cancer, additional genetic events are essential to malign transormation of most of anal carcinoma. Specialized studies did not explain already if this vírus is the cause or the associated factor to anal carcinoma. More research is needed to solve this doubt. Anyway, we suggest that clinic and sub-clinic lesions control could avoid the eventual evolution to invasive carcinoma.

Key words: Papillomavirus infections. Intra-epithelial neoplasia; prevention and control. Anal canal; cytology. Carcinoma, squamous cell.

\section{REFERÊNCIAS BIBLIOGRÁFICAS}

1. Papaconstantinou HT, Lee AJ, Simmang CL, Ashfaq R, Gokaslan ST, Sokol S, Huber PJ Jr, Gregorcyk SG. Screening methods for high-grade dysplasia in patients with anal condyloma. J Surg Res. 2005;127:8-13.

2. Palefsky JM, Holly EA, Efirdc JT, Da Costa M, Jay N, Berry JM, Darragh TM. Anal intraepithelial neoplasia in the highly active antiretroviral therapy era among HIV-positive men who have sex with men. AIDS. 2005;19:1407-14.

3. Piketty C, Kazatchkine MD.Human papillomavirus-related cervical and anal disease in HIV-infected individuals in the era of highly active antiretroviral therapy. Curr HIV/AIDS Rep. 2005;2:140-5.

4. Martin F, Bower M Anal intraepithelial neoplasia in HIV positive people. Sex Transm Infect. 2001;77(5):327-31.

5. Scholefield JH, Castle MT, Watson NF. Malignant transformation of high-grade anal intraepithelial neoplasia. Br J Surg. 2005;92(9):1133-6.

6. Gagne SE, Jensen R, Polvi A, Da Costa M, Ginzinger D, Efird JT, Holly EA, Darragh T, Palefsky JM. High-resolution analysis of genomic alterations and human papillomavirus integration in anal intraepithelial neoplasia. J Acquir Immune Defic Syndr. 2005;40:182-9. 
7. Nadal SR, Calore EE, Manzione CR, Assakawa MA, Felix LM, Horta SHC. Incidência de neoplasias intraepiteliais anais em doentes HIV-positivos portadores de condilomas acuminados comparando períodos anterior e posterior aos inibidores da protease. Rev bras Coloproct 2005;25(3):217-22.

8. Fox PA. Human papillomavirus and anal intraepithelial neoplasia. Curr Opin Infect Dis. 2006;19(1):62-6.

9. Chin-Hong PV, Vittinghoff E, Cranston RD, Browne L, Buchbinder S, Colfax G, Da Costa M, Darragh T, Benet DJ, Judson F, Koblin B, Mayer KH, Palefsky JM. Age-related prevalence of anal cancer precursors in homosexual men: the EXPLORE study. J Natl Cancer Inst. 2005;97:896-905.

10. Manzione CR, Nadal SR, Calore EE. Postoperative followup of anal condylomata acuminata in $\mathrm{HIV}+$ patients. Dis Colon Rectum, 2003;46(10):1358-1365.

11. Fagan SP, Bellows CF 3rd, Albo D, Rodriquez-Barradas M, Feanny M, Awad SS, Berger DH. Length of human immunodeficiency virus disease and not immune status is a risk factor for development of anal carcinoma. Am J Surg. 2005;190(5):732-5.

12. Daling JR, Madeleine MM, Johnson LG, Schwartz SM, Shera KA, Wurscher MA, Carter JJ, Porter PL, Galloway DA, McDougall JK. Human papillomavirus, smoking, and sexual practices in the etiology of anal cancer. Cancer. 2004;101(2):270-80.

13. Gervaz P, Hahnloser D, Wolff BG, Anderson SA, Cunningham J, Beart RW Jr, Klipfel A, Burgart L, Thibodeau SN. Molecular biology of squamous cell carcinoma of the anus: a comparison of HIV-positive and HIV-negative patients. J Gastrointest Surg. 2004;8:1024-30.

14. Bower M, Palmieri C, Dhillon T. AIDS-related malignancies: changing epidemiology and the impact of highly active antiretroviral therapy. Curr Opin Infect Dis. 2006;19(1):14-9.

15. Varnai AD, Bollmann M, Griefingholt H, Speich N, Schmitt C, Bollmann R, Decker D. HPV in anal squamous cell carcinoma and anal intraepithelial neoplasia (AIN) Impact of HPV analysis of anal lesions on diagnosis and prognosis. Int $\mathbf{J}$ Colorectal Dis. 2006;21(2):135-42.

16. Gonzalez-Bosquet E, Almagro MM, Mora I, Sunol M, Callejo J, Lailla JM. Prevalence of human papilloma virus infection of the uterine cervix in women with abnormal cervical cytology. Eur J Gynaecol Oncol. 2006;27(2):135-8.

17. de Carvalho JJ, Syrjanen KJ, Jacobino M, Rosa NT, Carvalho LZ. Prevalence of genital human papillomavirus infections established using different diagnostic techniques among males attending a urological clinic. Scand J Urol Nephrol. 2006;40(2):138-43.

18. Guillaud M, Adler-Storthz K, Malpica A, Staerkel G, Matisic J, Van Niekirk D, Cox D, Poulin N, Follen M, Macaulay C. Subvisual chromatin changes in cervical epithelium measured by texture image analysis and correlated with HPV. Gynecol Oncol. 2005;99(3 Suppl 1):S16-23.
19. Manzione CR, Nadal SR, Calore EE. Oncogenicidade do Papilomavirus humano e o grau de neoplasia intraepitelial anal em doentes HIV positivo. Rev Assoc Med bras 2004;50(3): 282-5.

20. Vandepapeliere P, Barrasso R, Meijer CJ, Walboomers JM, Wettendorff M, Stanberry LR, Lacey CJ. Randomized controlled trial of an adjuvanted human papillomavirus (HPV) type 6 L2E7 vaccine: infection of external anogenital warts with multiple HPV types and failure of therapeutic vaccination. J Infect Dis. 2005;192(12):2099-107

21. Nadal SR, Calore EE, Nadal LRM, Horta SHC, Manzione CR. Citologia anal para rastreamento de leões pré-neoplásicas. Rev bras Coloproct 2005;25(supl.):63.

22. Palefsky JM, Holly EA, Hogeboom CJ, Berry JM, Jay N, Darragh TM. Anal cytology as a screening tool for anal squamous intraepithelial lesions. J Acquir Immune Defic Syndr Hum Retrovirol 1997;14:415-22.

23. Arain S, Walts AE, Thomas P, Bose S. The Anal Pap Smear: Cytomorphology of squamous intraepithelial lesions. Cytojournal. 2005;2:4.

24. Fox PA, Seet JE, Stebbing J, Francis N, Barton SE, Strauss S et al. The value of anal cytology and human papillomavirus typing in the detection of anal intraepithelial neoplasia: a review of cases from an anoscopy clinic. Sex Transm Infect. 2005;81:142-6.

25. Cranston RD, Darragh TM, Holly EA, Jay N, Berry JM, Costa MD et al. Self-Collected Versus Clinician-Collected Anal Cytology Specimens to Diagnose Anal Intraepithelial Neoplasia in HIV-Positive Men. J Acquir Immune Defic Syndr. 2004; 36:915-920.

26. Durante AJ, Williams AB, Da Costa M, Darragh TM, Khoshnood K, Palefsky JM. Incidence of anal cytological abnormalities in a cohort of human immunodeficiency virusinfected women. Cancer Epidemiol Biomarkers Prev. 2003; 12:638-42.

27. Devaraj B, Cosman BC. Expectant management of anal squamous dysplasia in patients with HIV. Dis Colon Rectum. 2006;49(1):36-40.

28. Gervaz P, Hirschel B, Morel P. Molecular biology of squamous cell carcinoma of the anus. Br J Surg. 2006;93(5):531-8.

29. Steenbergen RD, de Wilde J, Wilting SM, Brink AA, Snijders PJ, Meijer CJ. HPV-mediated transformation of the anogenital tract. J Clin Virol. 2005;32 Suppl 1:S25-33.

\section{Endereço para correspondência:}

\section{SIDNEY ROBERTO NADAL}

Rua Dr. Virgilio de Carvalho Pinto, 381 apt. 23

05415-030, São Paulo

Tel/ Fax (+ 55 11) 3337-4282

E-mail:srnadal@terra.com.br 